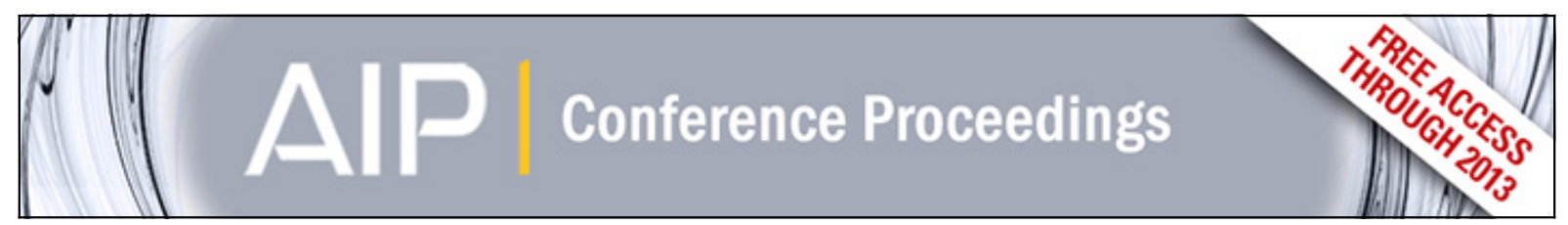

\title{
Glass transitions in monolayers of colloidal ellipsoids
}

Zhongyu Zheng and Yilong Han

Citation: AIP Conference Proceedings 1518, 153 (2013); doi: 10.1063/1.4794562

View online: http://dx.doi.org/10.1063/1.4794562

View Table of Contents: http://scitation.aip.org/content/aip/proceeding/aipcp/1518?ver=pdfcov

Published by the AIP Publishing 


\title{
Glass transitions in monolayers of colloidal ellipsoids
}

\author{
Zhongyu Zheng* and Yilong Han ${ }^{\dagger}$ \\ *Institute of mechanics, Chinese Academy of Sciences, Beijing, China. \\ ${ }^{\dagger}$ Department of Physics, The Hong Kong University of Science and Technology, Hong Kong, China
}

\begin{abstract}
Glass formers constitute of anisotropic particles was mainly studied by simulations in three dimensions with incomplete phase diagrams. Here we studied the structures and the glassy dynamics for translational and rotational motions in quasi-two dimensional (2D) suspensions of colloidal ellipsoids at the single-particle level. At high densities, ellipsoids with large aspect ratio formed psuedo-nematic domains. Video microscopy revealed a two-step glass transition: rotational motion first becomes glassy due to the inter-domain freezing, then translational motion become glassy at a higher density due to inner-domain freezing. Between the two transitions, ellipsoids formed an "orientational glass". Below and near the respective glass transition densities, the rotational and translational fastest-moving particles moved cooperatively and formed clusters with power-law size distributions. The mean cluster sizes diverge in power law as approaching the glass transitions. The fast translational particles concentrated in pseudonematic domains and form band-like clusters while the fast rotational particles mainly located around domain boundaries and form branch-like clusters.
\end{abstract}

Keywords: glass transition, colloid, ellipsoid

PACS: $64.70 . p v, 82.70 . \mathrm{Dd}$

\section{INTRODUCTION}

Glass transition is one of the deepest and most important unsolved problems in condensed matter physics. Colloids have been proved as outstanding model systems for glass transition studies because single-particle dynamics is measurable by video microscopy and image processing. To date, significant insights about glass transitions have been obtained from colloidal glasses composed of spheres [1]. Much less effort has been expended, however, to study of glasses composed of anisotropic particles. In fact, anisotropic colloidal particles can better model glasses which are usually composed of molecules with anisotropic shapes or interactions. The simulation and theoretical studies on glasses composed of anisotropic particles are also far less than enough and even the phase diagrams of some simple shaped anisotropic particles are not available. Molecular mode-coupling theory (MMCT) predicts a kinetic phase diagram of three-dimensional (3D) monodispersed hard ellipsoids of revolution as a function of packing fraction $\phi$ and aspect ratio $p[2,3]$. It predicts a rotator phase at small $p(>1)$, a one-step glass transition at intermediate $p$ and a two-step glass transitions at large $p$. However MMCT cannot predict nematic phase at very large $p$ and solid-liquid coexistence for hard spheres when $p \simeq 1$.

A few simulations have briefly explored the glassy dynamics of chains of hard spheres [4] and nucleation of short hard rods in 3D [5]. A recent simulation explored the dynamics around the glass transitions in 3D, but their ellipsoids have small aspect ratios $(p=1.25$ prolate and 0.8 oblate), hence the two-step glass transition with the orientational glass was not able to be observed [6]. The only simulation about phase behaviors of $2 \mathrm{D}$ ellipses focused on the nematic order in small systems with 200 ellipses [7]. Glass transitions for anisotropic particles in 2D have not been explored in theory, simulation or experiment.

Recently there are a few experiments studies on colloidal glasses composed of anisotropic particles, but the focuses are on their normal modes [8] and rheology properties [9] rather than the glass transition dynamics. To this end, we carried out experiments about glass transitions in colloidal glass constitute of ellipsoids under 2D confinements which have the following advantages: (1) It is one the the simplest glasses consisted of uniform sized particles. Note that monodispersed disks in $2 \mathrm{D}$ can only form crystals even at the fastest quenching rate. Hence bidispersed or highly polydispersed spheres have been used in experiments [10, 11, 12], simulations [13] and theory [14] for 2D glasses. In contrast, we found that monodispersed ellipsoids of intermediate aspect ratio are excellent glass formers in 2D because their shape can effectively frustrate crystallization and nematic order. (2) The trajectories of all the particle in the region of interest can be tracked, which are difficult to be measured in three dimensional systems. (3) Rotational motion can be measured for ellipsoids, but not for spheres. We discovered that translational and rotational motions have different glass transition points with an intermediate "orientational glass" phase [15]. 


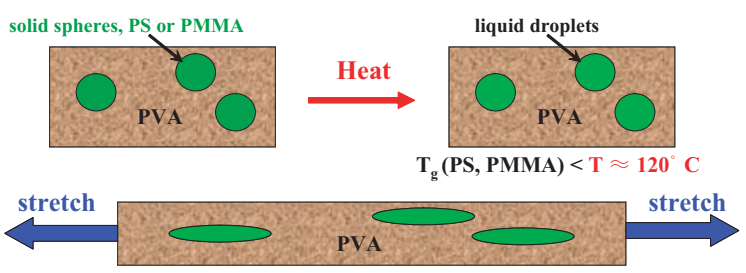

FIGURE 1. Making ellipsoids by stretching spheres.

\section{SAMPLE PREPARATION AND IMAGE ANALYSIS}

The ellipsoids were synthesized by stretching polymethyl methacrylate (PMMA) spheres [16, 17], see Fig. 1. PMMA spheres were added into an aqueous polyvinyl alcohol (PVA) solution (12\% weight fraction) in a Petri dish. After water was evaporated at room temperature, the solid PVA film was stretched at $130^{\circ} \mathrm{C}$ which is above the glass transition temperatures of PVA $\left(T_{g}=85^{\circ} \mathrm{C}\right)$ and PMMA $\left(T_{g}=105^{\circ} \mathrm{C}\right)$ so that the PMMA spheres could be deformed into ellipsoids. After cooling to room temperature, the PVA was dissolved and washed for more than five times in deionized water and ellipsoids were obtained. Since ellipsoids are stretched from spheres with uniform surface charge densities, ellipsoids' tips are expected to have higher surface charge density than the waist, but quantitative charge distribution is unknown. Nevertheless, the charge on ellipsoid should be effectively screened out due to the $\mathrm{Na}^{+}$ions released from glass walls in such thin sample with high surfaceto-volume ratio and the $3 \mathrm{mM}$ sodium dodecyl sulfate (SDS) added to stabilize ellipsoids. The total ionic strength is more than $3 \mathrm{mM}$ and the corresponding Debye length is less than $20 \mathrm{~nm}$. Hence ellipsoids can be considered as moderately hard particles. Isolated ellipsoids in a dilute monolayer can be well fitted by 2D Gaussian functions with semi-long axis $a$ and semi-short axis $b$ as fitting parameters [18]. The two batches of ellipsoids are used: (1) aspect ratio $p=6$ with the semi-long axis $a=3.33 \mu \mathrm{m}$ and the semi-short axes $b=c=0.56 \mu \mathrm{m}$; (2) aspect ratio $p=9$ with the semi-long axis $a=x x x \mu \mathrm{m}$ and the semi-short axes $b=c=0 . x x \mu \mathrm{m}$. From the variance of fitted $a$ for $\sim 200$ particles, we found that the polydispersity of the long axis is $5.6 \%$. In the paper, we will mainly use the data about ellipsoids with $p=6$ to illustrate most of the results.

To prepare the 2D sample, a tiny droplet of $0.5 \mu \mathrm{L}$ suspension was placed on a glass slide and then a coverslip is placed on. The glass surfaces were rigorously cleaned so that the droplet can spread into a thin film by capillary force and particles did not stick to the surfaces. Then the $12 \times 15 \times 0.1 \mathrm{~mm}^{3}$ glass cell was sealed with epoxy glue. The area fraction $\phi \equiv \pi a b \rho$, where $\rho$ is the num-
(A)

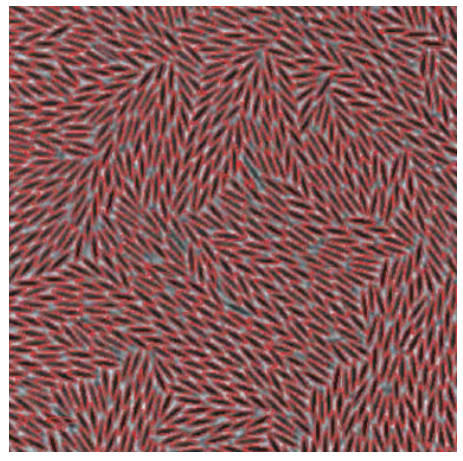

(B)

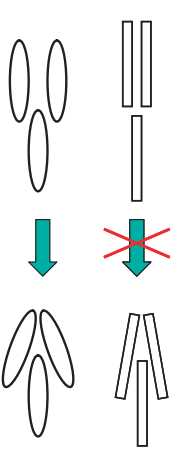

FIGURE 2. (A) A typical particle-tracking result of the $2 \mathrm{D}$ suspension of colloidal ellipsoids (the area fraction $\phi=0.70$ ). The red elliptical contours represent the positions and orientations of the ellipsoids obtained from image analysis, which coincide well with the dark images of the real ellipsoids. (B) In close packing, a third ellipsoid tends to insert into the two neighbors and change their orientations, while rectangular rods tend to align with the same orientation.

ber density averaged over all video frames. $\phi$ was tuned by placing the cells vertically for particles to slowly drift under gravity for several hours to one day. When the desired area fraction was reached in the $0.01 \mathrm{~mm}^{2}$ chosen area, the sample was placed horizontally for 2-3 hours for equilibration. The area fraction and the correlations are almost the same for 2-hour and 12-hour equilibration, thus the systems are in metastable equilibrium without obvious ageing effect.

We measured the central $0.01 \mathrm{~mm}^{2}$ area for 3 to 6 hours at each density. Light interference measurements showed that the wall separation varied by only $\sim 30 \mathrm{~nm}$ per $1 \mathrm{~mm}$ [17], so the walls could be considered as parallel within the field of view. The fluctuation in the $\mathrm{z}$ direction appeared to be very weak and these heavy ellipsoids always stayed in the focal plane. The area fraction remained a constant during the whole observation period. We measured 3-5 densities in one sample cell and totally 12 densities in three cells. The wall separation is a constant for different densities in one cell because we chose a fixed observation area. The wall separations in different cells were all about $1.5 \mu \mathrm{m}$ from the particle diffusion measurements and the interference color measurements shown in ref. [19]. The particle tips occasionally go above or below each other, which delays glass transitions to $\sim 2 \%$ higher area fractions. For brevity, we nevertheless use 2D instead of quasi-2D. Twelve densities were measured in the range $0.20 \leq \phi \leq 0.81$. During the three to six hours measurments at each $\phi$, no drift flow or density change was observed.

The thermal motion of the ellipsoids was recorded using a charge-coupled device camera resolving $1392 \times 1040$ pixels at 1 frame per second (fps) for 
the highest five concentrations and at $3 \mathrm{fps}$ for lower concentrations. The center-of-mass positions and orientations of individual ellipsoids were tracked using our image processing algorithm [19], see the result in Fig. 2a. The first step in the image processing algorithm is to apply a spatial band-pass filter to remove the noises in the image [20], then use the standard sphere-tracking algorithm [20] to identify the position of ellipsoids. However, one ellipsoid might be incorrectly tracked as multiple spheres, i.e. multiple candidate positions of the center of mass. The second step is to remove the wrong candidate positions and roughly estimated the true position of each ellipsoid in a $4 a \times 4 a$ area centered around each position. We created $184 a \times 4 a$ masks; each mask contains an ellipsoid with orientation $0^{\circ}, 10^{\circ}, \cdots$, or $170^{\circ}$, then found the largest total brightness of (mask $\times$ area) among the 18 total brightnesses. If the largest total brightness was greater than a empirical critical value, it was considered as an ellipsoid. In step three, the position and orientation of ellipsoid obtained from step two is further refined. The area was scanned pixel by pixel and $1^{\circ}$ by $1^{\circ}$ with a $4 a \times 4 b$ mask. The total brightness of (mask $\times$ area) was calculated at each position and orientation. Finally, we fit with a parabolic function and interpolate the position of the maximum brightness and obtain sub-pixel resolution. All ellipsoids in the field of view can be captured well with our algorithm, see the the image processing result shown by red ellipses in Fig. 2a. The major tracking error was from the image distortion due to the small tumbling motions in quasi-2D confinement. From the intercept of the mean-square displacement (MSD), we found the angular resolution was $1^{\circ}$ and the spatial resolutions were $0.12 \mu \mathrm{m}$ and 0.04 $\mu \mathrm{m}$ along the long and the short axes respectively. At high densities the ellipsoids spontaneously formed small pseudo-nematic domains with branch-like structures each involving about $10^{2}$ particles, see Fig. 2a. .

In some of the data analysis, we projected step displacements measured in fixed lab frame $\delta \mathbf{x}_{n}$ to the step displacement $\delta \tilde{\mathbf{x}}_{n}$ in the local body-frame attached on ellipsoid. As shown in Fig. 3 the two are related via a rotation, $\delta \tilde{x}_{n i}=\mathscr{R}_{i j} \delta x_{n j}$, where

$$
\mathscr{R}_{i j}=\left(\begin{array}{cc}
\cos \theta_{n} & \sin \theta_{n} \\
-\sin \theta_{n} & \cos \theta_{n}
\end{array}\right)
$$

is the rotation matrix with $\theta_{n}=\left(\theta\left(t_{n-1}\right)+\theta\left(t_{n}\right)\right) / 2$. In practice, choosing $\theta_{n}=\theta\left(t_{n-1}\right)$ or $\theta_{n}=\theta\left(t_{n}\right)$ has little affect on our results because $\theta$ barely changes in two consecutive frames. We can construct total body-frame displacements by summing over displacements in each step: $\tilde{\mathbf{x}}\left(t_{n}\right)=\sum_{k=1}^{n} \delta \tilde{\mathbf{x}}_{k}$, and from $\tilde{\mathbf{x}}\left(t_{n}\right)$, we can construct body-frame displacements for trajectories of duration $t$ at starting time $\tau_{0}$ via $\Delta \tilde{\mathbf{x}}(t)=\tilde{\mathbf{x}}\left(t+\tau_{0}\right)-\tilde{\mathbf{x}}\left(\tau_{0}\right)$ so that transverse and longitudinal motions can be separately tracked [18].

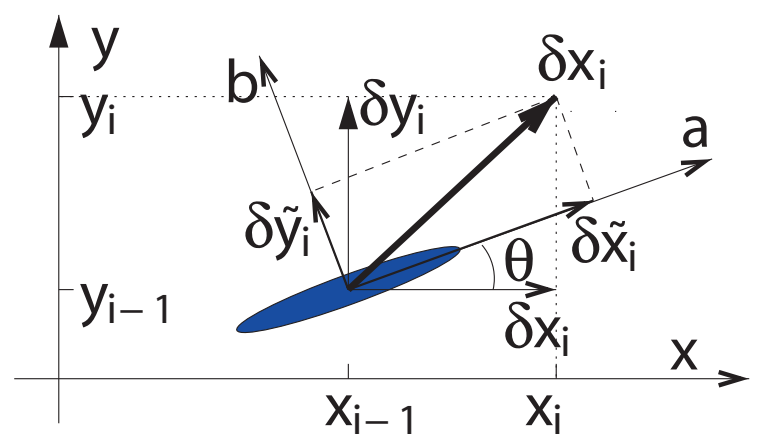

FIGURE 3. Transformation between the $x-y$ lab-frame displacements $(\delta x, \delta y)$ and the $\tilde{x}$ - $\tilde{y}$ body-frame displacements $(\delta \tilde{x}$, $\delta \tilde{y})$. The angle between two frames is $\theta(t)$.

\section{STATIC STRUCTURE}

We measure the static structures from the radial distribution function of center of mass $g^{T}(r)=\left\langle\rho\left(\mathbf{r}^{\prime}+\right.\right.$ $\left.\left.\mathbf{r}^{\prime}, t\right) \rho(\mathbf{r}, t)\right\rangle$ and the angular correlation function $g_{2}^{\theta}(r)=$ $\langle\cos (2[\theta(0)-\theta(r)])\rangle[7,21]$, see Fig. 4. Here $\rho(\mathbf{r})=$ $\sum_{i=1}^{N} \delta\left(\mathbf{r}-\mathbf{r}_{i}\right)$ is the density, $\theta(r)$ is the orientation of ellipsoid at distance $r$, and \langle\rangle is the ensemble and time average. $g_{2 \theta}(r) \equiv 1,0$ and -1 for parallel, random and perpendicular arrangement, respectively. In Fig. 4(a), the angular correlation increases with $\phi$. At high $\phi, g_{2}^{\theta}(r)$ exhibit exponential decay at large distances and algebraiclike decay at short distances, indicating random orientations across domains and quasi-nematic orders within domains. $g_{2 T}(r)$ develops a few peaks at $r=2.4 b, 4.2 b$ and $6 b$ at high densities, corresponding to the dense packing configurations of side-by-side $(r \simeq 2 b)$, side-totip $(r \simeq a+b)$ and tip-to-tip $(r \simeq 2 a=6 b)$. No obvious structural change was observed near the glass transition point.

We did not observed nematic phase or semetic domains found in 3D spherocylinders [5] because 1) the elliptical shape facilitates particles changing orientation and forming branch-like structures at high densities, while rectangular rods tends to keep the orientational order [22], see Fig. 2b; 2) The 5.6\% polydispersity promotes glass formation. 3) Long-wavelength fluctuations are stronger in $2 \mathrm{D}$ than in $3 \mathrm{D}$, which can more easily break the long-range order as described by MerminWagner theorem. Ellipsoids with $p \sim 6$ appeared to be good glass formers, which can easily preempt any isotropic-nematic (IN) phase transition [7]. In contrast, the glass transition can be preempted by crystallization for $p \simeq 1$ in $2 \mathrm{D}$, or by an IN transition for rods with $p>25$ in $3 \mathrm{D}$ [4]. 

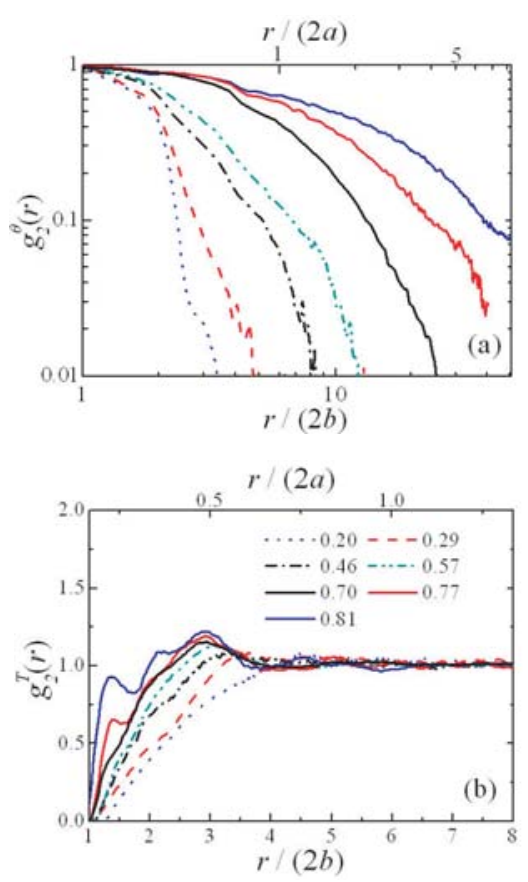

FIGURE 4. The angular correlation function $g_{2}^{\theta}(r)$ and (b) the radial distribution function $g_{2}^{T}(r)$ in the 2D suspensions of colloidal ellipsoids at different area fractions.

\section{DYNAMIC PROPERTIES}

\section{A. Structural relaxation}

The translational relaxation was characterized by the self-intermediate scattering function $F_{S}(q, t) \equiv\left\langle\sum_{j=1}^{N} e^{i \mathbf{q} \cdot\left(\mathbf{x}_{j}(t)-\mathbf{x}_{j}(0)\right)}\right\rangle / N \quad$ where $\quad \mathbf{x}_{j}(t) \quad$ is the position of ellipsoid $j$ at time $t, N$ is the total number of particles, $\mathbf{q}$ is the wave vector and \langle\rangle denotes a time average. In Fig. 5(a), we chose $q_{m}=2.3 \mu \mathrm{m}^{-1}$ measured from the first peak position in the structure factor at high density. At high $\phi$, a two-step relaxation is developed in $F_{S}(q, t)$ which is a signature of dynamic heterogeneity when the liquid is approaching the glass transition. The short-time $\beta$-relaxation corresponds to diffusion within cages formed by neighboring particles, and the long-time $\alpha$-relaxation reflects structural rearrangement involving a series of cage breaking. According to mode-coupling theory (MCT), $F_{s}(q, t)=f_{q}+h_{q} G(t)$ holds at the initial stage of the $\beta$-relaxation and $F_{s}(q, t)=f_{q}-h_{q} G(t)$ holds at the crossover time to the $\alpha$-relaxation respectively. $f_{q}$ is the plateau height, $h_{q}$ is the amplitude and $G(t)$ is a universal function called $\beta$ correlator independent to $q$. $F_{s}(q, t)$ decays as the critical-decay law $G(t) \sim t^{-a}$ at shorter time and the von Schweidler law $G(t) \sim t^{-b}$ at longer
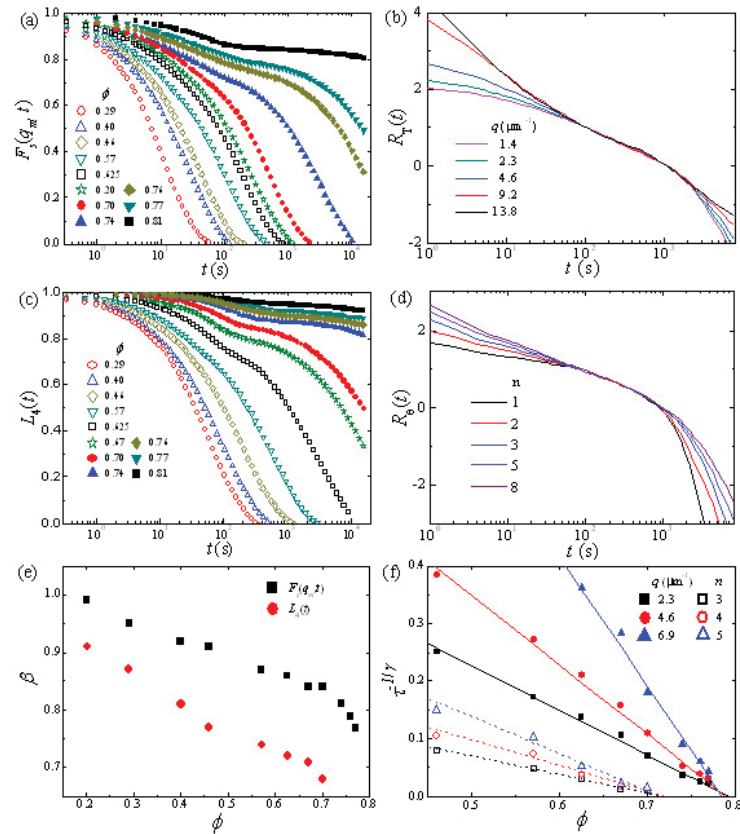

FIGURE 5. (a) The self-intermediate scattering function $F_{s}(q, t)$ at $q_{m}=2.3 \mu \mathrm{m}^{-1}$. (b) $R_{T}(t)=\left[F_{s}(q, t)-\right.$ $\left.F_{S}\left(q, t_{1}\right)\right] /\left[F_{S}\left(q, t_{2}\right)-F_{S}\left(q, t_{1}\right)\right]$ for different $q$ at $\phi=0.77$. (c) The orientational correlation $L_{4}(t)$ for different area fractions. (d) $R(t)=\left[L_{n}(t)-L_{n}\left(t_{1}\right)\right] /\left[L_{n}\left(t_{2}\right)-L_{n}\left(t_{1}\right)\right]$ for different order $n$ at $\phi=0.70$. Here $t_{1}$ and $t_{2}$ were chosen at $101 \mathrm{~s}$ and $1084 \mathrm{~s}$ in (a) and $76 \mathrm{~s}$ and $936 \mathrm{~s}$ in (b). (e) The exponent $\beta$ of the fitting function $e^{-(t / \tau)^{\beta}}$ for the long-time $F_{s}\left(q_{m}, t\right)$ and $L_{4}(t)$. (f) The fitted relaxation time $\tau(\phi) \sim\left(\phi_{c}-\phi\right)^{-\gamma}$. Solid symbols: different choices of $q$ in $F(q, t)$ for translational motions. Open symbols: different choices of $n$ in $L_{n}(t)$ for orientational motions.

time. The exponent $a$ and $b$ are independent of $\phi$ and $q$ and characterize a particular system. So, $F_{s}(q, t)-f_{q}$ can be separated or factorized into a $q$-dependent and a $t$-dependent part. We can cancel $q$ by defining $R_{T}(t) \equiv\left[F_{s}(q, t)-F_{s}\left(q, t_{1}\right)\right] /\left[F_{s}\left(q, t_{2}\right)-F_{s}\left(q, t_{1}\right)\right]=$ $\left[G(q, t)-G\left(q, t_{1}\right)\right] /\left[G\left(q, t_{2}\right)-G\left(q, t_{1}\right)\right]$, where $t_{1}$ and $t_{2}$ are two arbitrary times taken in the regime where the two power laws hold. Fig. 5b shows that $R_{T}(t)$ for different $q$ at $\phi=0.77$ collapse and keep ordered around the plateau region in $F_{S}(q, t)$, which confirms the validity of the MCT "factorization property" in the 2D colloidal ellipsoid suspensions. The "factorization property" has been observed in simulations of 3D Lennard-Jones particles [23] and 3D hard ellipsoids [6], but not in 2D systems before.

The rotational relaxation can be characterized by the orientational correlation function $L_{n}(t) \equiv\left\langle\sum_{j=1}^{N} \cos n\left(\theta_{j}(t)-\theta_{j}(0)\right)\right\rangle / N$ where $n$ is a positive integer and $\theta_{j}$ is the orientation of ellipsoid $j$. 
$L_{n}(t)$ decays faster for larger $n$, and different choices of $n$ can yield the same glass transition point. $n=4$ in Fig. $5 \mathrm{c}$ was chosen so that $L_{n}(t)$ can be better displayed within our measured time scales. Interestingly, the "factorization property" also exists in the rotational degrees of freedom. Figure 5d demonstrates that the intermediate regions in the rescaled orientational correlation functions $R(t)=\left[L_{n}(t)-L_{n}\left(t_{1}\right)\right] /\left[L_{n}\left(t_{2}\right)-L_{n}\left(t_{1}\right)\right]$ also collapse together for different order $n$ in deeply supercooled liquid. This scaling law for rotational motion has not been predicted in the MMCT $[2,24]$ or observed in experiments and simulations before. The factorization property indicates that a $t$-dependent part should be extracted from the $L_{n}(t)$ around the plateau regions independent of $\phi$ and $n$.

The $\alpha$-relaxations at different $\phi$ follow the stretchedexponential decay (Kohlrausch function) $e^{-(t / \tau)^{\beta}}$ where $\tau$ is the relaxation time. Figure 5e shows that the fitted exponent $\beta$ decreases from 0.92 to 0.77 for $F_{S}\left(q_{m}, t\right)$ and from 0.81 to 0.68 for $L_{4}(t)$ as $\phi$ increases from 0.40 towards the glass transition point. Lower value of $\beta$ corresponds to stronger dynamic heterogeneity, hence the system is more dynamically heterogeneous and the motions are more cooperative at higher $\phi$.

In MCT, $\tau(\phi)$ diverges algebraically as approaching the critical point $\phi_{c}: \tau(\phi) \sim\left(\phi_{c}-\phi\right)^{-\gamma}$ where $\gamma=$ $1 /(2 a)+1 /(2 b)[25]$. By fitting the critical-decay law and the von Schweidler law, we obtained $a_{T}=0.3 \pm 0.02$ and $b_{T}=0.63 \pm 0.02$ for $F_{S}\left(q_{m}, t\right)$ and $a_{\theta}=0.32 \pm 0.02$ and $b_{\theta}=0.65 \pm 0.02$ for $L_{4}(t)$. Consequently $\gamma_{T}=$ $2.45 \pm 0.05$ and $\gamma_{\theta}=2.33 \pm 0.05$ for the translational and orientational correlations respectively. These values are close to the $\gamma_{T}=2.3$ measured for 3D ellipsoids [6]. In Fig. 5f, $\tau^{-1 / \gamma}$ is linear in $\phi$ for different choices of $q$ and $n$. Interestingly, all the scalings show that the glass transitions are at $\phi_{c}^{\theta}=0.72 \pm 0.01$ for rotational motion and $\phi_{c}^{T}=0.79 \pm 0.01$ for translational motion. This indicates three distinct phases: liquid $(\phi<0.72)$, an intermediate orientational glass which is liquid-like in its translational degrees of freedom but glassy in its rotational degrees of freedom $(0.72<\phi<0.79)$, and the glass state for both degrees of freedom $(\phi>0.79)$.

\section{B. Diffusion and dynamic heterogeneity}

The diffusions are characterized by the translational and rotational mean-square displacements: $\operatorname{MSD}_{\|, \perp}(t) \equiv$ $\left\langle\Delta x_{\|, \perp}^{2}(t)\right\rangle$ along long- (II) and short- $(\perp)$ axes in the particle frame and rotational $\operatorname{MSD}_{\theta}(t) \equiv\left\langle\Delta \theta^{2}(t)\right\rangle$, see Figs. 6a,b. The initial increase in MSDs reflects shorttime diffusion inside cages. The plateau in the intermediate time scale indicates that the motion is hindered by the caging of neighboring particles. The rise up of the
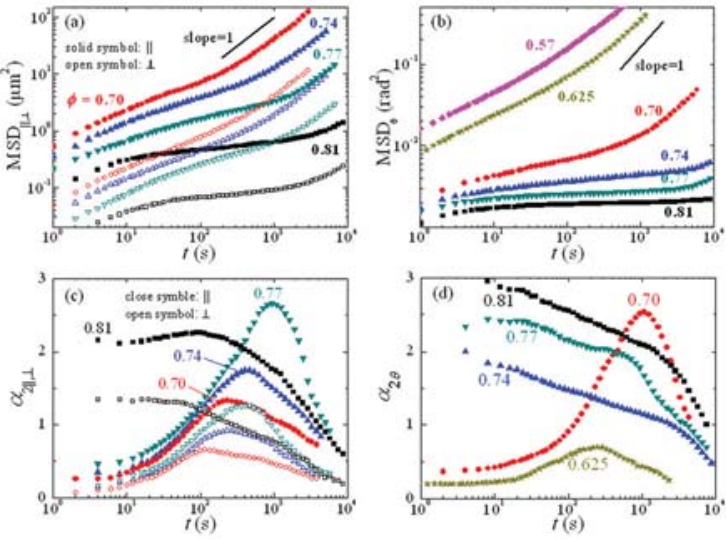

FIGURE 6. (a) The translational MSDs along long- (II) and short- $(\perp)$ axes. (b) The rotational MSDs. The non-Gaussian parameters of translational displacements along the long axis $\left(\alpha_{2}^{\|}(t)\right.$, solid symbols) and the short axis $\left(\alpha_{2}^{\perp}(t)\right.$, open symbols). $\phi=0.70,0.74,0.77,0.81$ as labeled in the figures. (b) The non-Gaussian parameters of rotational displacements.

plateau corresponds to cage breaking in the $\beta$-relaxation and then crosses over to a long-time diffusion due to the cage rearrangement. At high density, the plateau became significantly flat and stretched which are the characteristics of glass [26, 27], and its long-time rise up corresponds to the activated hopping processes [28]. MSD is much larger than $\mathrm{MSD}_{\perp}$ for all densities and time intervals, which indicates that motion along long axes are dominate in both the short-time in-cage rattling and the long-time caging breaking.

As approaching the glass transition, the dynamics not only dramatically slow down but also become progressively more heterogeneous, i.e. some regions may exhibit much faster dynamics than others even though, spatially, these regions may be very close. For heterogeneous dynamics, the step-size distribution is strongly non-Gaussian. The degree of non-Gaussian behavior is measured by the non-Gaussian parameters $\alpha_{2}(t)=$ $\left\langle\Delta x^{4}\right\rangle /\left(3\left\langle\Delta x^{2}\right\rangle^{2}\right)-1$ of particle displacements $\Delta x$ during time $t$ [26]. A distribution is Gaussian when $\alpha_{2}=0$ and non-Gaussian when $\alpha_{2}>0$. In supercooled liquids, particle motion is diffusive before it reaches its cage and after many cage-breaking rearrangements. Hence the distribution of $\Delta x$ is Gaussian at short and long times. At the intermediate times, however, it becomes non-Gaussian with long tails due to cooperative out-of-cage displacements [26, 27, 29]. It can be observed from the chains of long arrows in Fig. 7 about the translational displacements. Such cooperative motion is strongest at time $\tau_{2}$ corresponding to the peak of $\alpha_{2}(t)$, see Fig. 6. As $\phi$ increases, the peak rises and shifts towards a longer time, indicating growing dynamic heterogeneity and slowing 


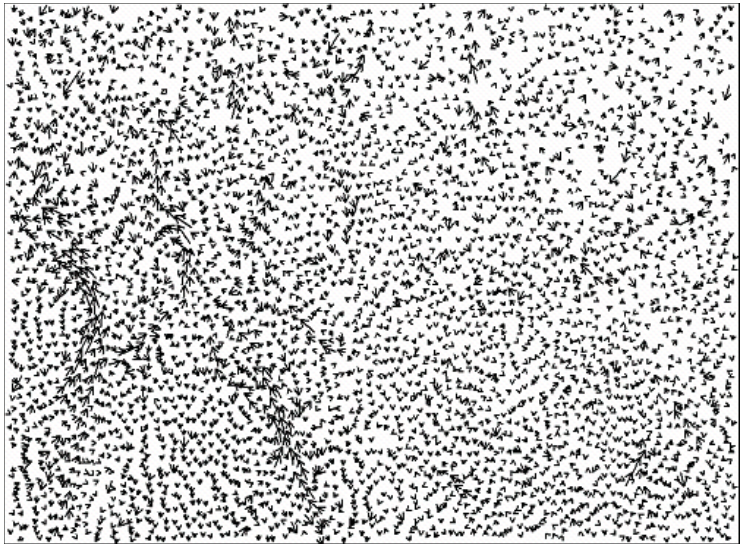

FIGURE 7. The cooperative motions represented by the translational displacement vectors of ellipsoids during a $250 \mathrm{~s}$ time interval at the area fraction $\phi=0.70$. The length of the black lines denote the displacements of particles The red and black dots at the two ends of each line represent the initial and final positions of the centers of mass.

down dynamics respectively on approaching the glass transitions. In contrast, the glass phase lacks cooperative out-of-cage motions and all particles are caged, so $\alpha_{2}(t)$ exhibits no distinct peak and declines with time [26]. Such a sharp change has been regarded as a characteristic of a glass transition [26]. Figure 6 clearly shows the glass transitions at $\phi_{c}^{\theta}=0.72 \pm 0.02$ for rotational motion and at $\phi_{c}^{T}=0.79 \pm 0.02$ for translational motion.

We also measured the long-time diffusion coefficients for translational and rotational motions and compared them with the relaxation times. Relaxation and diffusion were decoupled in both translational and rotational motions. The ratio of the translational and rotational diffusion coefficients deviates from a constant, which indicates that the Stocks-Einstein (SE) relation and the Stocks-Einstein-Debye (SED) relation breaks down at different degrees of supercooling. SE or SED relation is a form of the fluctuation-dissipation theorem (FDT) [30]. The breakdown of SE relation can be explained from the coupling model based on the fact that $\beta$ is larger for translational diffusion than rotation $[31,32]$ as we observed in Fig. 5e. Similar breakdown of SE has been observed in hard spheres [30] and breakdown of SED has been observed in a mixture of ellipsoids and spheres [33].

\section{Spatial distribution of fastest particles}

The dynamic heterogeneity can be directly visualized from the spatial distribution of the fastest-moving particles [26], see Fig. 7. Colored particles in Fig. 8 further show the distribution of the fastest-moving $8 \%$ of the particles in supercooled liquid $(\phi=0.70)$, orientational glass $(\phi=0.77)$ and double glass $(\phi=0.81)$. The time intervals are chosen as $\tau_{2}$ for $\phi<\phi_{c}$ and as the middle time of MSDs plateaus at $\phi>\phi_{c} .8 \%$ particles are chosen because the non-Gaussian long tail of the distribution of $\Delta x\left(\tau_{2}\right)$ covers about $8 \%$ of the population. Different choices of $t$ and the percentage yield the similar results. Neighboring fastest-moving ellipsoids form clusters and are labeled using the same color. Here two ellipsoids are defined as neighbors if they overlap after being expanded by 1.5 times and their closest distance does not intersect a third particle. In the supercooled liquid, most fast particles were strongly spatially correlated and formed large extended clusters, see Fig. 8. This demonstrates the $\alpha$-relaxation occurs by cooperative particle motion in both the translational and rotational degrees of freedom: when one particle moves, another particle moves closely following the first. The colloidal glasses, in contrast, show no discernible $\alpha$-relaxation, and the fastest particles in $\beta$-relaxation are randomly dispersed without forming large clusters [26], as observed in the 3D glass transition of colloidal spheres [26]. Figure 8 clearly depicts three regimes: both the translational and rotational fast particles are distributed heterogeneously with large clusters at $\phi<0.72$; the rotational fast particles are dispersed homogenously while the translational fast particles form large clusters at $0.72<\phi<0.79$; and both types of fast particles are dispersed homogenously at $\phi>0.79$.

The spatial distributions of translational and rotational fast-particle clusters were anticorrelated. The fast translational tended to gather in the pseudonematic domains to form band-like clusters, while the fast rotational particles mainly located in orientational-disordered regions between these domains to form branch-like clusters. It is sensible that the nematic orders within a domain facilitate the cooperative translation along the domain orientation but hinder the rotational motion, while the orientational disorders near boundaries ease the cooperative rotation but restrain the translational motion. As increased towards the rotational and translational glass transitions respectively, slow and fast particles more and more concentrated together to form a few larger clusters indicating stronger rotational and translational heterogeneities. All the phases in Fig. 8 contain some isolated fast translational and rotational particles; they are mainly distributed at the domain boundaries with random orientations. In contrast to the observed anti-correlations between fast translational and rotational motions, the simulations of supercooled liquids of modeled water with $p \simeq 1.4$ [?] and dumbbells with $p \simeq 1.8$ [?], in which the pseudonematic domains are absent, showed coupled translational and rotational relaxations with a one-step glass transition. Hence the dynamic heterougeneity strongly depend on the structure which is largely dictated by particle 


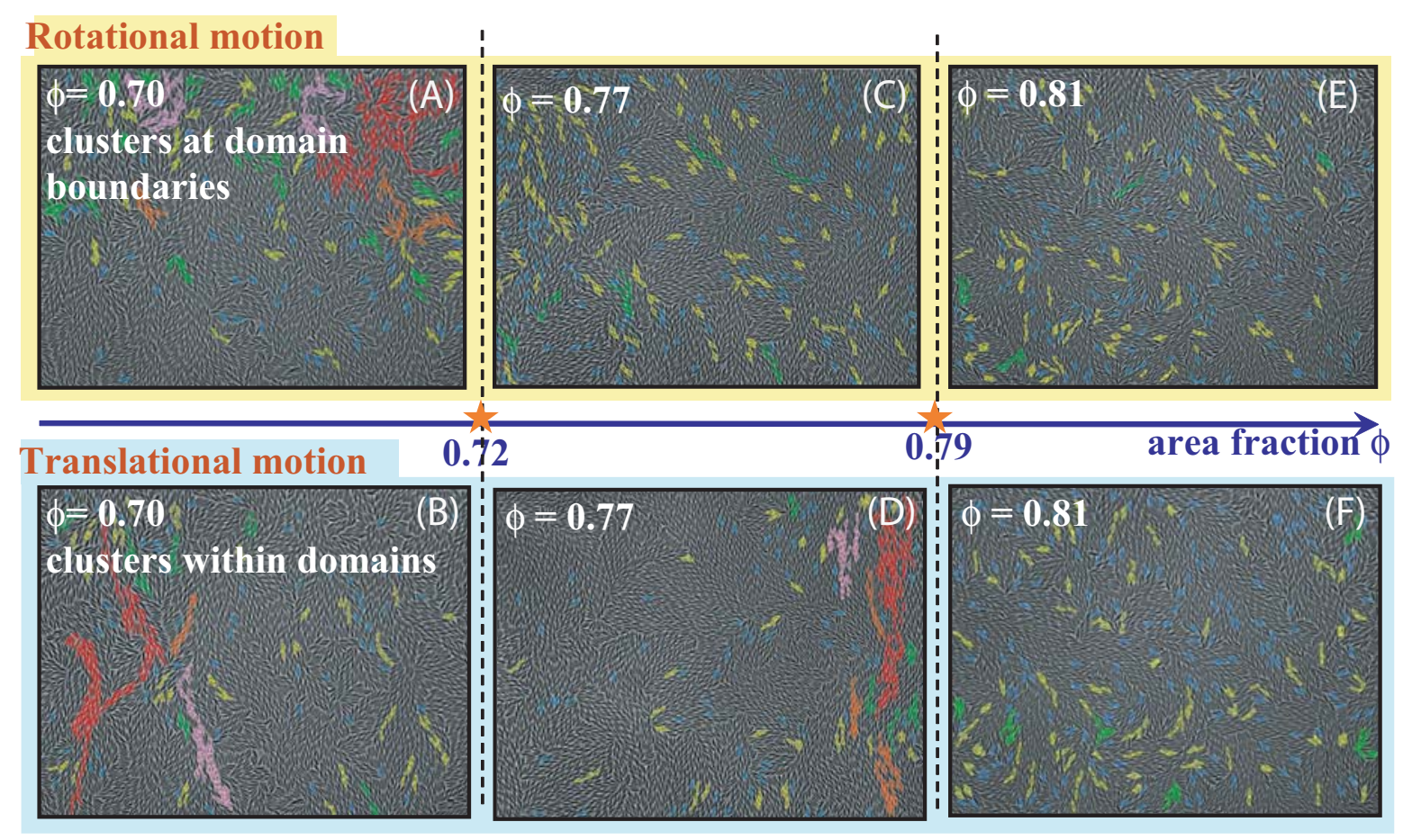

FIGURE 8. The spatial distributions of the fastest-moving $8 \%$ of the particles (labeled in colors) in translational (a, c, e) and rotational (b, d, f) motions. Ellipsoids in the same cluster have the same color. (a, b) The same frame at $\phi=0.70$ (supercooled liquid); (c, d) The same frame at $\phi=0.77$ (orientational glass); (e, f) The same frame at $\phi=0.81$ (double glass) with $\sim 5500$ particles.

shape.

The cluster sizes of the fast particles, $N_{c}$, exhibit a power-law distribution $P\left(N_{c}\right) \sim N_{c}^{-\mu}$ as shown in Figs. 9a, b. The fitted exponents $\mu$ for translational and rotational motions change dramatically near their respective glass transitions see Fig. 9c. The $\mu^{\theta, T}=2.0 \pm 0.2$ for supersaturated liquids is close to the $\mu^{T}=2.2 \pm 0.2$ estimated for hard spheres [26] and the $\mu^{T}=1.9 \pm$ 0.1 for Lennard-Jones particles in 3D [29], while the $\mu^{\theta, T}=3.2 \pm 0.1$ for glasses is close to the $\mu^{T}=3.1$ estimated for hard spheres in 3D [26]. Hence $\mu \simeq 2.5$ might characterize such glass transitions in general. Figure $9 \mathrm{~d}$ shows the weighted mean cluster size $\left\langle N_{c}\right\rangle=$ $\sum N_{c}^{2} P\left(N_{c}\right) / \sum N_{c} P\left(N_{c}\right)[26,29]$ at different densities. Both $\left\langle N_{c}^{\theta}\right\rangle$ and $\left\langle N_{c}^{T}\right\rangle$ diverge on approaching the corresponding $\phi_{c}:\left\langle N_{c}\right\rangle \sim\left(\phi_{c}-\phi\right)^{-\eta}$ with fitted $\eta^{\theta}=0.81$ and $\eta^{T}=0.75$, indicating growing cooperative regions of mobile particles and corresponding to increasing dynamic heterogeneity. Similar scaling and $\eta^{T}$ have been observed in a Lennard-Jones system [29], but the mechanism is not clear.
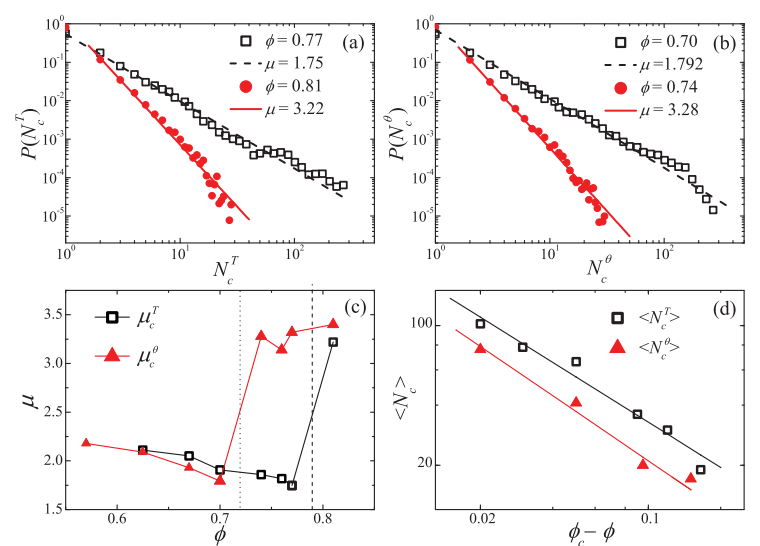

FIGURE 9. The probability distribution functions for the cluster size of (a) translational and (b) rotational fastest-moving particles. The lines are the best fits of $P\left(N_{c}\right) \sim N_{c}^{-\mu}$. (c) The fitted exponents $\mu^{\theta}$ for rotational motions and $\mu^{T}$ for translational motions. The vertical dotted and dashed lines represent the glass transitions for rotational and translational motions respectively. (d) The weighted mean cluster size $\left\langle N_{c}\right\rangle \sim$ $\left(\phi_{c}-\phi\right)^{-\eta}$ where $\phi_{c}^{\theta}=0.71$ and $\phi_{c}^{T}=0.79$. 


\section{SUMMARY AND DISCUSSION}

We conclude that a monolayer of colloidal ellipsoids with aspect ratio around 6 to 9 exhibit two glass transitions with an intermediate orientational glass. The two glass transitions in the rotational and translational degrees of freedom correspond to inter-domain freezing and inner-domain freezing respectively. The nematic order within a domain facilitates translational relaxation while the orientational disorder near domain boundaries promotes rotational relaxation. Fast translational particles are responsible for the out-of-cage diffusion, while fast rotational particles are responsible for domain transformations such as splitting, merging and rotating. All of the measurements consistently showed that the glass transitions for ellipsoids with $p=6$ confined between two walls are at $\phi_{c}^{\theta}=0.72$ for rotational motion and at $\phi_{c}^{T}=0.79$ for translational motion. For longer ellipsoids with $p=9(a=5.9 \mu \mathrm{m}, b=c=0.65 \mu \mathrm{m})$, $\phi_{c}^{\theta}=0.64 \pm 0.02$ and $\phi_{c}^{T}=0.73 \pm 0.02$ were observed in the two-wall confinement. This suggests that the intermediate regime between $\phi_{c}^{\theta}$ and $\phi_{c}^{T}$ increases with the aspect ratio. When aspect ratio becomes very large, however, we can expect that glass transitions gives the way to nematic transition even though nematic phase cannot be predicted by MMCT. When aspect ratio is small, ellipsoids cannot form psuedo-nematic domains. Consequently the translational and rotational motion should become glassy at the same point without the orientational glass phase. When aspect ratio is very close to 1, ellipsoids should pack into crystals as 2D hard disks but with random orientational order, i.e. rotator phase.

Approaching the glass transitions, the structural relaxation time and the mean cluster size for cooperative motion diverge, a typical feature of a glass transition $[34,25,29]$. Interestingly, the translational and orientational cooperative motions are anticorrelated in space, which has not been predicted in theory or simulation. A similar two-step glass transition has been observed in a 3D liquid-crystal system and explained as the freezing of the orientations of the pseudo-nematic domains and the freezing of the translational motion within domains [35]. Here we directly observed the conjectured pseudo-nematic domains in ref. [35]. These results at single-particle resolution shed new light on the formation of molecular glasses, especially at low dimensionality.

Ellipsoids provide aspect ratio as a tuning parameter to explore various effects on fragility, structure and dynamic heterogeneity. In particular, how does dynamics heterogeneity couple with static structure is an important question, but the effect is often too subtle to be detected. We observed several novel connections between structure and the dynamic heterogeneity as they becomes prominent when the aspect ratio $p>2.5$. Glass without psuedo-nematic domains at small $p$ and orientational glass with psuedo-nematic domains at large $p$ exhibit remarkable opposite behaviors, but also share some common features. These results will be reported in the future paper.

\section{ACKNOWLEDGMENTS}

We thank Maurizio Nobili and Ning $\mathrm{Xu}$ for the helpful discussion. The present study was financially supported by the grant RPC11SC04, F-HK32/10T and PHC PROCORE Nř 25265WL.

\section{REFERENCES}

1. G. Hunter, and E. Weeks, Rep. Prog. Phys. 75, 066501 (2012).

2. R. Schilling, and T. Scheidsteger, Phys. Rev. E 56, 2932-2949 (1997).

3. M. Letz, R. Schilling, and A. Latz, Phys. Rev. E 62 , 5173-5178 (2000).

4. G. Yatsenko, and K. Schweizer, Langmuir 24, 7474-7484 (2008).

5. R. Ni, S. Belli, R. van Roij, and M. Dijkstra, Phys. Rev. Lett. 105, 088302 (2010).

6. P. Pfleiderer, K. Milinkovic, and T. Schilling, Europhys. Lett. 84, 16003 (2008).

7. J. A. Cuesta, and D. Frenkel, Phys. Rev. A 42, 2126-2136 (1990).

8. P. Yunker, Z. Zhang, and A. G. Yodh, Phys. Rev. Lett. 104, 15701 (2010).

9. R. C. Kramb, R. Zhang, K. S. Schweizer, and C. F. Zukoski, Phys. Rev. Lett. 105, 055702 (2010).

10. H. König, R. Hund, K. Zahn, and G. Maret, Eur. Phys. J. E 18, 287-293 (2005).

11. Z. Zhang, N. Xu, D. T. Chen, P. Yunker, A. M. Alsayed, K. B. Aptowicz, P. Habdas, A. J. Liu, S. R. Nagel, and A. G. Yodh, Nature 459, 230-233 (2009).

12. P. Yunker, Z. Zhang, K. B. Aptowicz, and A. G. Yodh, Phys. Rev. Lett. 103, 115701 (2009).

13. R. Speedy, J. Chem. Phys. 110, 4559 (1999).

14. M. Bayer, J. M. Brader, F. Ebert, M. Fuchs, E. Lange, G. Maret, R. Schilling, M. Sperl, and J. P. Wittmer, Phys. Rev. E 76, 011508 (2007).

15. Z. Zheng, F. Wang, and Y. Han, Phys. Rev. Lett. 107, 65702 (2011).

16. C. Ho, A. Keller, J. Odell, and R. Ottewill, Colloid. Polym. Sci. 271, 469-479 (1993).

17. Y. Han, A. Alsayed, M. Nobili, and A. G. Yodh, Phys. Rev. E 80, 011403 (2009).

18. Y. Han, A. Alsayed, M. Nobili, J. Zhang, T. Lubensky, and A. Yodh, Science 314, 626-630 (2006).

19. Z. Zheng, and Y. Han, J. Chem. Phys. 133, 124509 (2010).

20. J. C. Crocker, and D. G. Grier, J. Colloid Interface Sci. 179, 298-310 (1996).

21. T. Kirchhoff, H. Löwen, and R. Klein, Physical Review E 53, 5011 (1996).

22. V. Narayan, N. Menon, and S. Ramaswamy, J. Stat. Mech. Theor. Exp. p. P01005 (2006).

23. T. Gleim, and W. Kob, Eur. Phys. J. B 13, 83-86 (2000). 
24. T. Franosch, M. Fuchs, W. Götze, M. R. Mayr, and A. P. Singh, Phys. Rev. E 56, 5659-5674 (1997).

25. W. Gotze, and L. Sjogren, Rep. Prog. Phys. 55, 241 (1992).

26. E. R. Weeks, J. C. Crocker, A. C. Levitt, A. Schofield, and D. A. Weitz, Science 287, 627 (2000).

27. W. Kegel, et al., Science 287, 290 (2000).

28. C. Angell, Journal of Physics and Chemistry of Solids 49 , 863-871 (1988).

29. C. Donati, S. C. Glotzer, P. H. Poole, W. Kob, and S. J. Plimpton, Phys. Rev. E 60, 3107-3119 (1999).

30. D. Bonn, and W. Kegel, J. Chem. Phys. 118, 2005 (2003).

31. K. Ngai, J. Phys. Chem. B 103, 10684-10694 (1999).

32. K. Ngai, Philos. Mag. 87, 357-370 (2007).

33. D. Chakrabarti, and B. Bagchi, Phys. Rev. Lett. 96, 187801 (2006)

34. W. Götze, and L. Sjögren, Phys. Rev. A 43, 5442-5448 (1991).

35. H. Cang, J. Li, V. Novikov, and M. Fayer, J. Chem. Phys. 119, 10421 (2003). 